\title{
Development of a Model for Predicting the Effectiveness of Pulsed Radiofrequency on Zoster- Associated Pain
}

\author{
Zhiyou Peng · Jianguo Guo · Yanfeng Zhang · Xuejiao Guo • \\ Wenguang Huang · Yunze Li · Zhe Yan · Nannan Guo • \\ Daqiang Ke $\cdot$ Li Chen · Jinyan Huang $\cdot$ Zhiying Feng (D)
}

Received: November 11, 2021 / Accepted: January 17, 2022 / Published online: January 30, 2022

(c) The Author(s) 2022

\begin{abstract}
Introduction: Zoster-associated pain (ZAP), which may cause anxiety, depression, and sleep disorders and reduce quality of life, is often refractory to current standard treatments. Studies have shown that pulsed radiofrequency (PRF) can alleviate ZAP and reduce the incidence of postherpetic neuralgia (PHN). This study aimed to explore the clinical characteristics associated with PRF responsiveness, develop a model for identifying risk factors of inadequate PRF management, and help clinicians make better decisions.

Methods: Patients who underwent PRF for ZAP between January 2017 and October 2020 in our hospital were included in this study. Patients were evaluated using the numerical rating scale
\end{abstract}

Z. Peng · J. Guo - Y. Zhang · X. Guo - W. Huang ·

Y. Li · Z. Yan · N. Guo · D. Ke · Z. Feng $(\bowtie)$

Department of Pain Medicine, College of Medicine, the First Affiliated Hospital, Zhejiang University, \#79 Qingchun Road, Hangzhou 310003, Zhejiang, China

e-mail: fzy1972@zju.edu.cn

L. Chen · J. Huang

Biomedical Big Data Center, the First Affiliated Hospital, Zhejiang University School of Medicine, 79 Qingchun Road, Hangzhou 310003, Zhejiang, China

J. Huang

Zhejiang University Cancer Center, Zhejiang

University, Hangzhou, China
(NRS), Insomnia Severity Index, Patient Health Questionnaire-9, and 36-Item Short Form Health Survey (SF-36) before and 3 months after the procedure. Patient demographic data and blood test results were also collected. We defined the effectiveness of PRF for ZAP as relief of $>50 \%$ in NRS scores compared to pre-PRF. Least absolute shrinkage and selection operator (LASSO) regression analyses were subsequently performed to identify factors related to the therapeutic effect of PRF in patients with ZAP. The performance of the prediction model was assessed by the area under the receiver operating characteristic curve (AUC).

Results: The effectiveness of PRF in patients with ZAP was $69.6 \%$ (total 313 patients) after 3 months. LASSO regression analysis extracted the seven most powerful features in the developed prediction model: sex, stage of herpes zoster (HZ), pregabalin dose, bodily pain indicators of SF-36, lymphocyte count, and low-density lipoprotein cholesterol (LDLC) and complement C4 in peripheral blood. Model $=1.586+0.148$ $\times$ lymphocyte $+(-0.001) \times$ bodily pain indicators of SF-36 $+(-0.001) \times$ pregabalin dose + $0.028 \times \mathrm{LDLC}+0.001 \times \mathrm{C} 4+(-0.508) \times \operatorname{sex}$ $+(-0.128) \times$ stage of HZ. We generated the ROC curve for the prediction model, and the final AUC was 0.701 . The sensitivity, specificity, and overall accuracy of the model were 90\%, 33\%, and $73 \%$, respectively.

Conclusions: Seven factors were significantly associated with poor PRF outcome: male sex, 
advanced stage of $\mathrm{HZ}$, higher pregabalin dose, higher bodily pain indicators of SF-36, and lower lymphocyte count, LDLC, and complement $\mathrm{C} 4$ in the peripheral blood. PRF should be applied to patients with ZAP as early as possible to achieve satisfactory outcomes.

Keywords: Zoster-associated pain; LASSO regression; Postherpetic neuralgia; Pulsed radiofrequency

\section{Key Summary Points}

Zoster-associated pain (ZAP) may cause physical disability and psychological depression that substantially impair patients' quality of life.

Pulsed radiofrequency (PRF) has recently been used to treat ZAP, offering the advantage of pain control without tissue destruction.

The factors that affect the patient's responsiveness to PRF treatment are still unclear. A statistical model for predicting ZAP was obtained through machine learning by LASSO regression.

Our result showed that seven factors were significantly associated with poor PRF outcome: male sex, advanced stage of herpes zoster, higher pregabalin dose, higher bodily pain indicators of SF-36, and lower lymphocyte count, low-density lipoprotein cholesterol, and complement $\mathrm{C} 4$ in the peripheral blood.

PRF should be applied to patients with ZAP as early as possible to achieve satisfactory outcomes. Patients at high risk of poor response to PRF treatment are advised to undergo further pain intervention as soon as possible.

\section{INTRODUCTION}

Herpes zoster (HZ) is a viral infection resulting from the reactivation of varicella-zoster virus (VZV) among the T lymphocytes in the dorsal root ganglions in patients with low cell-mediated immunity due to advanced age, immunosuppressive medical conditions, and malignancies, with a lifetime incidence of 30\% [1]. Zoster-associated pain (ZAP) is an extremely important clinical feature in patients with HZ, and pain may persist for months or even years, manifested as postherpetic neuralgia (PHN). Although pain in some patients with $\mathrm{HZ}$ is relieved by routine treatment, including antiviral agents, antidepressants, and anticonvulsants, in many patients the condition still progresses to PHN. PHN is conventionally defined as chronic pain persisting at least 90 days after the appearance of acute $\mathrm{HZ}$ rash. It is now widely accepted that the pathogenesis of PHN is central sensitization caused by nerve inflammation, increased nerve excitability, and change in neural plasticity [2]. PHN develops in $20-50 \%$ of patients with $\mathrm{HZ}$ and can lead to several months of treatment and reduced quality of life [3]. A study showed that the annual incidence of $\mathrm{HZ}$ in China is approximately $7.7 \%$. Of patients with $\mathrm{HZ}, 29.8 \%$ developed $\mathrm{PHN}$, indicating that there are approximately 2 million patients with PHN for every 100 million Chinese individuals [4]. Adults aged $>40$ years who live with PHN in a large urban center in China were characterized by a burden that was not limited to pain and pain interference with daily activities but also included poor sleep and low health-related quality of life, indicating that PHN is associated with a substantial patient and economic burden in China [5]. To date, therapy has been aimed at relieving the pain and symptoms secondary to PHN [6].

Pulsed radiofrequency (PRF) is a standard therapeutic strategy with a modification of thermal radiofrequency treatment. PRF has recently been used to treat ZAP, offering the advantage of pain control without tissue destruction. Moreover, the effects of PRF on ZAP lasted $>3$ months, and PRF improved the quality of life and sleep, without major 
complications [1]. Although it was also shown that the phase of diseases, degree of skin lesion, and depression are independent risk factors, whereas hypertension is a potential protective factor for developing analgesic resistance among patients with ZAP, the factors that affect patient responsiveness to PRF are still unclear. To improve the effects of PRF and help clinicians make better decisions, this study analyzed the influencing factors in ZAP treatment.

\section{METHODS}

\section{Study Design and Participants}

All subjects in this retrospective study provided written informed consent for their participation, and the process was approved by the research ethics committee of the Department of Pain Management at the First Affiliated Hospital, School of Medicine, Zhejiang University (IIT2021116). The subjects comprised patients with ZAP who underwent PRF and met the inclusion and exclusion criteria for this study, conducted between January 2017 and October 2020 .

The inclusion criteria were as follows: (1) age $>40$ years; (2) location of pain consistent with the HZ region and pain originating from HZ; (3) moderate to severe pain with numerical rating scale (NRS) score $\geq 4$ before enrollment after routine treatment, such as anticonvulsants, antidepressants, opioids, or physical treatments, for 2 weeks.

The exclusion criteria were as follows: cognitive deficits or mental disorders affecting the patient's treatment or questionnaire evaluation, serious cardio-cerebrovascular disease, hepatorenal insufficiency, infectious diseases, and other contraindications for PRF. Patients who were lost to follow-up for 3 months after PRF were also excluded from the present analysis. If the effect of PRF was not satisfactory, we adjusted the drug treatment strategy or further adopted intrathecal morphine infusion system implantation or spinal cord electrical stimulation at the appropriate time.

\section{Study Protocol and Procedure}

After admission, blood examinations were performed, including routine blood test, blood sedimentation, hypersensitive C-reactive protein, blood lipids, liver function, kidney function, immunoglobulin and complement, and tumor markers. All patients received orally administered drugs (gabapentin/pregabalin/duloxetine, nonsteroidal anti-inflammatory drugs [NSAIDs], and antiviral drugs). Dermatome corresponding to the region of $\mathrm{HZ}$ infection or PHN was identified if hypersensitivity and pain occurred in the corresponding HZ-affected region.

After patients were placed in an appropriate position with electrocardiographic monitoring and oxygen inhalation, they were treated with PRF (Baylis PM230 PRF generator, Baylis Medical Company, Inc., Montreal, Canada) using 18-G, straight, sharp radiofrequency cannulas with a 10-mm exposed tip, which were carefully inserted until the needle tip reached the upper edge of the intervertebral foramen. The guide methods used in the procedure were ultrasound, computed tomography (CT), radiography, ultrasound and CT, and ultrasound and radiography. After confirming the needle position with fluoroscopy guidance and sensory stimulation, the position of the needle tip was adjusted until the movement and sensation determined that the affected area was completely covered. The radiofrequency stimulation tests determined whether the $\mathrm{HZ}$ area was covered, as follows: (1) $50 \mathrm{~Hz}, 0.3-0.5 \mathrm{~V}$ test sensation, to induce pain sensation in the $\mathrm{HZ}$ area, and (2) $2 \mathrm{~Hz}, 0.3-0.5 \mathrm{~V}$ test exercise, in which pulse was induced when the nerve segment was affected. Standard PRF was conducted for $600 \mathrm{~s}$ at $42{ }^{\circ} \mathrm{C}$ sequentially. All patients returned to the ward after treatments, followed by a $15-\mathrm{min}$ observation period for complaints of discomfort. All PRF procedures were conducted by experienced physicians in our department.

\section{Data Collection}

The following data were collected from the medical records: sex, age, body mass index, 
Table 1 Basic statistics (pre-PRF) of the numerical variables

\begin{tabular}{|c|c|c|c|c|}
\hline & Variable & $\begin{array}{l}\text { Positive } \\
(\text { mean } \pm S D)\end{array}$ & $\begin{array}{l}\text { Negative } \\
(\text { mean } \pm S D)\end{array}$ & $P$ value \\
\hline \multirow{4}{*}{$\begin{array}{l}\text { Patient demographic } \\
\text { data }\end{array}$} & Age & $65.1 \pm 10.4$ & $66.8 \pm 10.4$ & 0.17 \\
\hline & Body mass index & $23.2 \pm 3.9$ & $24.0 \pm 5.0$ & 0.21 \\
\hline & Gabapentin dose (mg) & $443.6 \pm 579.4$ & $477.1 \pm 759.8$ & 0.70 \\
\hline & Pregabalin dose (mg) & $77.8 \pm 103.0$ & $117.7 \pm 173.2$ & 0.04 \\
\hline \multirow[t]{8}{*}{ Pain assessment scale } & Numerical rating scale & $4.8 \pm 1.9$ & $4.8 \pm 1.9$ & 0.85 \\
\hline & Times of breakthrough pain daily & $8.0 \pm 14.5$ & $13.2 \pm 28.9$ & 0.25 \\
\hline & Breakthrough pain score & $6.0 \pm 2.6$ & $6.8 \pm 2.8$ & 0.09 \\
\hline & Allodynia score & $4.5 \pm 2.9$ & $4.8 \pm 3.1$ & 0.41 \\
\hline & Numbness score & $3.2 \pm 2.9$ & $3.0 \pm 2.9$ & 0.66 \\
\hline & Insomnia Severity Index & $5.3 \pm 3.0$ & $5.4 \pm 2.9$ & 0.81 \\
\hline & ID pain & $3.3 \pm 1.2$ & $3.1 \pm 1.2$ & 0.43 \\
\hline & Douleur Neuropathique 4 & $5.0 \pm 2.0$ & $4.9 \pm 1.9$ & 0.50 \\
\hline \multicolumn{2}{|c|}{ Patient Health Questionnaire (PHQ)-9 } & $8.8 \pm 6.3$ & $7.6 \pm 5.7$ & 0.12 \\
\hline \multicolumn{2}{|c|}{ Generalized Anxiety Disorder (GAD)-7 } & $5.4 \pm 5.3$ & $4.8 \pm 5.3$ & 0.34 \\
\hline \multirow{7}{*}{$\begin{array}{l}\text { Brief Pain Inventory } \\
\quad(\mathrm{BPI})\end{array}$} & General activity & $5.3 \pm 2.8$ & $5.5 \pm 2.8$ & 0.59 \\
\hline & Mood & $5.0 \pm 3.0$ & $5.0 \pm 2.9$ & 0.94 \\
\hline & Walking ability & $3.4 \pm 2.8$ & $3.4 \pm 3.1$ & 0.88 \\
\hline & Normal work & $5.1 \pm 3.2$ & $5.2 \pm 3.7$ & 0.91 \\
\hline & Relations with other people & $2.9 \pm 2.8$ & $3.1 \pm 3.1$ & 0.76 \\
\hline & Sleep & $5.6 \pm 3.1$ & $5.7 \pm 3.1$ & 0.89 \\
\hline & Enjoyment of life & $4.3 \pm 3.0$ & $5.1 \pm 6.3$ & 0.25 \\
\hline \multirow[t]{9}{*}{ SF-36 } & Physical functioning & $63.2 \pm 25.8$ & $63.6 \pm 29.1$ & 0.91 \\
\hline & Role-physical & $24.0 \pm 39.9$ & $30.5 \pm 38.4$ & 0.19 \\
\hline & Bodily pain & $36.6 \pm 22.0$ & $43.0 \pm 21.5$ & 0.02 \\
\hline & General health & $47.8 \pm 18.4$ & $49.5 \pm 18.5$ & 0.48 \\
\hline & Vitality & $51.4 \pm 20.8$ & $54.5 \pm 21.0$ & 0.27 \\
\hline & Social functioning & $56.0 \pm 26.5$ & $62.7 \pm 27.5$ & 0.06 \\
\hline & Role-emotional & $28.4 \pm 40.6$ & $25.6 \pm 39.5$ & 0.43 \\
\hline & Mental health & $56.7 \pm 20.0$ & $59.4 \pm 19.3$ & 0.29 \\
\hline & Reported health transition & $2.4 \pm 1.2$ & $2.4 \pm 1.0$ & 0.78 \\
\hline
\end{tabular}


Table 1 continued

\begin{tabular}{|c|c|c|c|c|}
\hline & Variable & $\begin{array}{l}\text { Positive } \\
(\text { mean } \pm \text { SD })\end{array}$ & $\begin{array}{l}\text { Negative } \\
(\text { mean } \pm \text { SD })\end{array}$ & $P$ value \\
\hline \multirow[t]{8}{*}{ Routine blood test } & Platelet $\left(10^{9} / \mathrm{L}\right)$ & $215.3 \pm 64.7$ & $201.5 \pm 63.0$ & 0.08 \\
\hline & Hemoglobin $(\mathrm{g} / \mathrm{L})$ & $136.9 \pm 14.1$ & $133.6 \pm 19.3$ & 0.14 \\
\hline & Monocytes $\left(10^{9} / \mathrm{L}\right)$ & $0.42 \pm 0.17$ & $0.42 \pm 0.18$ & 0.61 \\
\hline & Eosinophil $\left(10^{9} / \mathrm{L}\right)$ & $0.027 \pm 0.015$ & $0.025 \pm 0.013$ & 0.44 \\
\hline & Granulocyte $\left(10^{9} / \mathrm{L}\right)$ & $4.03 \pm 1.83$ & $4.2 \pm 1.8$ & 0.55 \\
\hline & Basophil $\left(10^{9} / \mathrm{L}\right)$ & $0.14 \pm 0.14$ & $0.13 \pm 0.13$ & 0.81 \\
\hline & White blood cell $\left(10^{9} / \mathrm{L}\right)$ & $6.45 \pm 2.10$ & $6.36 \pm 1.93$ & 0.70 \\
\hline & Lymphocyte $\left(10^{9} / \mathrm{L}\right)$ & $1.84 \pm 0.73$ & $1.60 \pm 0.56$ & $<0.01$ \\
\hline \multirow[t]{10}{*}{ Biochemical } & $\begin{array}{l}\text { High-density lipoprotein cholesterol } \\
(\mathrm{mmol} / \mathrm{L})\end{array}$ & $1.21 \pm 0.33$ & $1.21 \pm 0.42$ & 0.90 \\
\hline & Triglycerides $(\mathrm{mmol} / \mathrm{L})$ & $1.77 \pm 1.21$ & $1.64 \pm 0.93$ & 0.34 \\
\hline & Cholesterol $(\mathrm{mmol} / \mathrm{L})$ & $4.81 \pm 1.19$ & $4.61 \pm 1.01$ & 0.18 \\
\hline & Very low-density lipoprotein $(\mathrm{mmol} / \mathrm{L})$ & $0.74 \pm 0.42$ & $0.71 \pm 0.34$ & 0.45 \\
\hline & Alanine aminotransferase $(\mathrm{U} / \mathrm{L})$ & $21.1 \pm 14.3$ & $21.0 \pm 12.7$ & 0.93 \\
\hline & Albumin $(\mathrm{g} / \mathrm{L})$ & $42.34 \pm 4.27$ & $41.70 \pm 5.18$ & 0.32 \\
\hline & Glucose $(\mathrm{mmol} / \mathrm{L})$ & $5.53 \pm 1.56$ & $5.63 \pm 1.66$ & 0.64 \\
\hline & Aspartate aminotransferase (U/L) & $20.46 \pm 7.52$ & $19.10 \pm 5.25$ & 0.08 \\
\hline & $\begin{array}{l}\text { Low-density lipoprotein cholesterol } \\
(\mathrm{mmol} / \mathrm{L})\end{array}$ & $2.85 \pm 0.95$ & $2.70 \pm 0.81$ & 0.19 \\
\hline & Creatinine $(\mathrm{mL} / \mathrm{min})$ & $85.0 \pm 17.4$ & $83.3 \pm 17.9$ & 0.44 \\
\hline \multirow[t]{6}{*}{ Tumor markers } & Carbohydrate antigen $19-9(\mathrm{U} / \mathrm{mL})$ & $9.43 \pm 13.5$ & $12.2 \pm 27.6$ & 0.41 \\
\hline & Carcinoembryonic antigen $(\mathrm{ng} / \mathrm{mL})$ & $2.66 \pm 1.65$ & $2.97 \pm 1.99$ & 0.22 \\
\hline & Alpha-fetoprotein $(\mathrm{ng} / \mathrm{mL})$ & $2.80 \pm 1.24$ & $2.62 \pm 1.39$ & 0.33 \\
\hline & Ferritin $(\mathrm{U} / \mathrm{mL})$ & $237.0 \pm 164.5$ & $304.7 \pm 294.3$ & 0.06 \\
\hline & Carbohydrate antigen $125(\mathrm{U} / \mathrm{mL})$ & $15.52 \pm 13.38$ & $18.93 \pm 18.65$ & 0.15 \\
\hline & Total prostate-specific antigen $(\mathrm{ng} / \mathrm{mL})$ & $1.21 \pm 1.50$ & $1.46 \pm 1.53$ & 0.38 \\
\hline \multirow[t]{3}{*}{ Immunoglobulin } & Immunoglobulin $\mathrm{G}(\mathrm{mg} / \mathrm{dL})$ & $1154.5 \pm 300.5$ & $1080.5 \pm 369.2$ & 0.17 \\
\hline & Immunoglobulin A (mg/dL) & $243.5 \pm 99.9$ & $240.7 \pm 141.9$ & 0.89 \\
\hline & Immunoglobulin $\mathrm{M}$ (mg/dL) & $98.9 \pm 65.1$ & $105.2 \pm 59.3$ & 0.50 \\
\hline \multirow[t]{2}{*}{ Complement } & Complement C3 (mg/dL) & $111.3 \pm 18.1$ & $108.3 \pm 19.5$ & 0.31 \\
\hline & Complement C4 (mg/dL) & $24.8 \pm 7.4$ & $23.7 \pm 6.9$ & 0.29 \\
\hline \multicolumn{2}{|c|}{ Erythrocyte sedimentation rate $(\mathrm{ESR})(\mathrm{mm} / \mathrm{h})$} & $13.3 \pm 12.3$ & $16.6 \pm 17.4$ & 0.13 \\
\hline
\end{tabular}


Table 1 continued

\begin{tabular}{cccc}
\hline Variable & $\begin{array}{l}\text { Positive } \\
(\text { mean } \pm \text { SD })\end{array}$ & $\begin{array}{l}\text { Negative } \\
(\text { mean } \pm \text { SD })\end{array}$ & $\boldsymbol{P}$ value \\
\hline C-reactive protein $(\mathrm{CRP})(\mathrm{mg} / \mathrm{L})$ & $5.6 \pm 13.9$ & $8.9 \pm 20.8$ & 0.23 \\
\hline
\end{tabular}

Mean the average of all samples, $S D$ standard deviation

education level, guide methods of the procedure, hypertension, diabetes, other complications (1, brain; 2 , heart; 3 , lung; 4, liver; 5 , kidney; 6 , tumor), rash site, days from $\mathrm{HZ}$ onset to PRF, and targeted nerve segment.

The assessment scales for pain and its concomitant symptoms were collected before and 3 months after the procedure, as follows: NRS, Brief Pain Inventory (BPI), Pain Catastrophizing Scale, Insomnia Severity Index, ID Pain, Generalized Anxiety Disorder-7, Patient Health Questionnaire-9, and Health Survey-36 (SF-36). BPI contains seven sub-items, including general activity, mood, walking ability, normal work, relations with other people, sleep, and enjoyment of life. SF-36 contains nine sub-items, including physical functioning, role-physical, bodily pain, general health, vitality, social functioning, role-emotional, mental health, and reported health transition. Doses of anticonvulsants (gabapentin mg/dL, pregabalin mg/ dL) and antiviral drugs, neurotropic drugs, opiates, and NSAIDs before PRF were also recorded.

\section{Data Processing}

For ease of calculation, we conducted the following data preprocessing: In terms of sex, we marked " 1 " for female and " 2 " for male. The guide methods of the procedure include ultrasound recorded as " 1 ," CT + ultrasound recorded as " 2, " radiography + ultrasound recorded as " 3 ," CT recorded as "4," and radiography recorded as "5." As for education level, we recorded illiteracy as "1," elementary school level as "2," junior high school as " 3 ," senior high school as " 4 ," and university and above as "5." We divided different stages of HZ into three layers, which were represented by consecutive numbers: $\leq 1$ month was recorded as " 1, " between 1 and 3 months was recorded as "2," and $>3$ months was recorded as " 3 ." As for rash site, we recorded the cervical segment as " 1 ," head segments as "2," lumbosacral segments as " 3 ," and thoracic segment as "4." For the remaining features, we denoted "no" as " 0 " and "yes" as "1." To describe effectiveness of the PRF treatment, we marked the negative as " 0 " and the positive as " 1 " according to general practices [7].

\section{Statistical Analysis}

The primary effectiveness endpoint was relief of $>50 \%$ in pain intensity as measured from baseline to 3 months after PRF, using an 11-point NRS. The outcome was effectiveness of the PRF treatment, which was defined as either "positive" or "negative."

To compare the difference between the two outcomes to select significant features, we used the $t$-test for continuous variables and chisquare tests for categorical variables. Correlation analysis was applied to select strongly correlated variables. The least absolute shrinkage and selection operator (LASSO) method is suitable for reducing high-dimensional data and is applied to select the most useful prediction candidates, so LASSO regression was used to construct the model with the previously selected variables. We used tenfold cross-validation to obtain the optimal regularization parameter, i.e., lambda, by minimizing the sum of the least square plus shrinkage penalty. LASSO regression was performed using the glmnet package in $\mathrm{R}$ version 4.1.1. The performance of the prediction model was assessed by the area under the receiver operating characteristic (ROC) curve (AUC). 
Table 2 Basic statistics (pre-PRF) of the categorical variables

\begin{tabular}{|c|c|c|c|}
\hline Variable & Category & Positive $(n)$ & Negative $(n)$ \\
\hline \multirow[t]{2}{*}{ Sex } & Female & 116 & 39 \\
\hline & Male & 102 & 56 \\
\hline \multirow[t]{5}{*}{ Guiding methods of procedure } & Ultrasound & 146 & 69 \\
\hline & $\mathrm{CT}+$ ultrasound & 26 & 4 \\
\hline & $\mathrm{X}$-ray + ultrasound & 23 & 6 \\
\hline & $\mathrm{CT}$ & 18 & 15 \\
\hline & $\mathrm{X}$-ray & 5 & 1 \\
\hline \multirow[t]{5}{*}{ Education level } & Illiteracy & 21 & 19 \\
\hline & Elementary school level & 28 & 8 \\
\hline & Junior high school & 70 & 32 \\
\hline & Senior high school & 78 & 25 \\
\hline & University and above & 21 & 11 \\
\hline \multirow[t]{6}{*}{ Concomitant disease } & Hypertension & & \\
\hline & No & 140 & 58 \\
\hline & Yes & 78 & 37 \\
\hline & Diabetes & & \\
\hline & No & 184 & 78 \\
\hline & Yes & 33 & 17 \\
\hline \multirow[t]{3}{*}{ Stage of herpes zoster } & $\leq 1$ month & 118 & 30 \\
\hline & 1-3 months & 64 & 28 \\
\hline & $\geq 3$ months & 35 & 37 \\
\hline \multirow[t]{2}{*}{ Left or right side of body } & Left & 108 & 49 \\
\hline & Right & 110 & 46 \\
\hline \multirow[t]{4}{*}{ Rash site } & Cervical & 30 & 13 \\
\hline & Head & 32 & 24 \\
\hline & Lumbosacral & 29 & 9 \\
\hline & Thoracic & 127 & 49 \\
\hline
\end{tabular}

\section{RESULTS}

A total of 313 participants were included in this study. Of these, $218(69.6 \%)$ patients had relief of pain after PRF, whereas 95 (30.4\%) were not responsive to PRF treatment. A total of 72 indicators were recorded for monitoring the status of the participants. The basic statistics of the numerical and categorical variables are shown in Tables 1 and 2, respectively. 
Lymphocyte

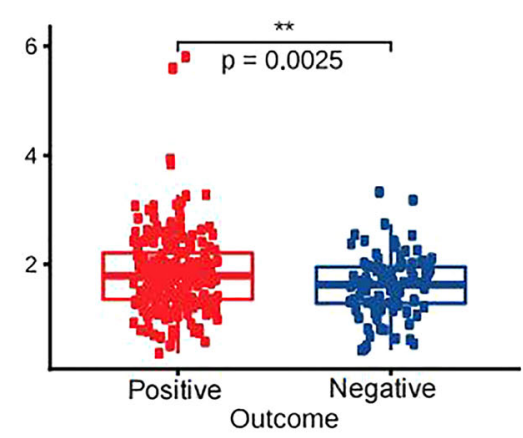

Bodily Pain indicators of SF-36

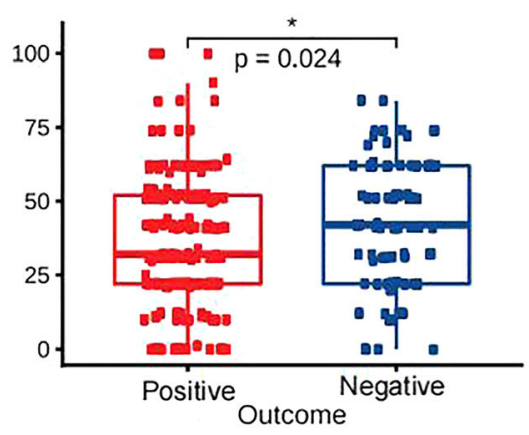

Pregabalin dose $(\mathrm{mg})$

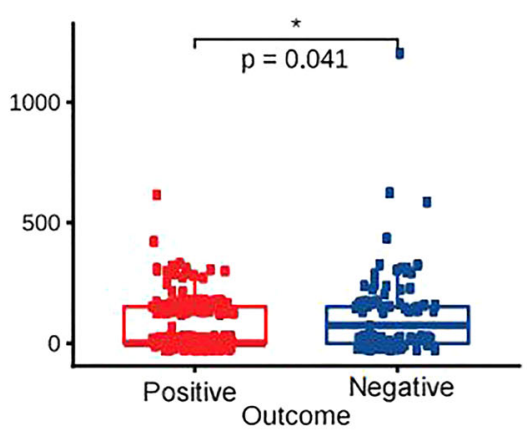

Fig. 1 Boxplot of the statistically significant variables
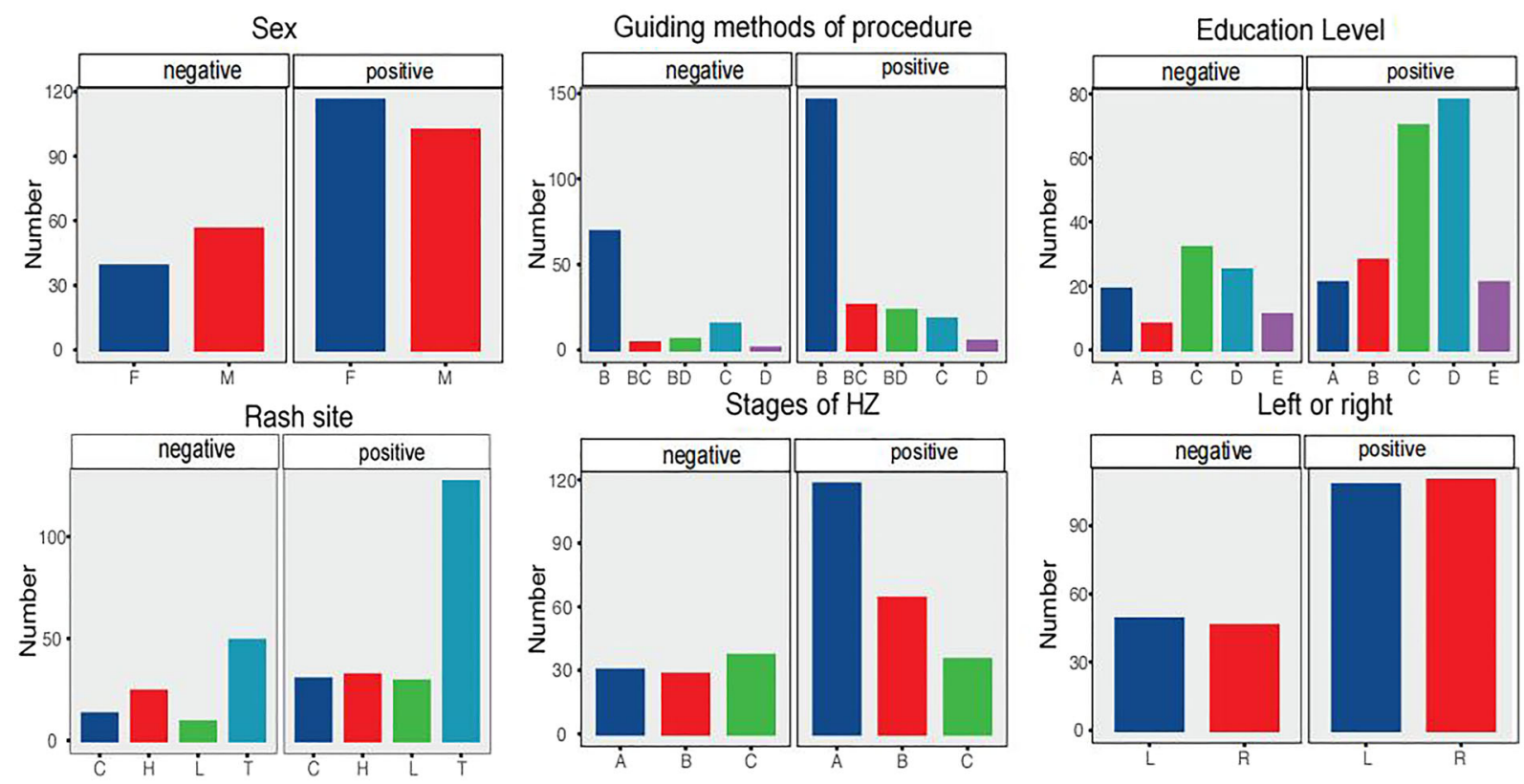

Fig. 2 Barplot of categorical variables. Sex: $F$ female, $M$ male. Guide methods of procedure: $B$ ultrasound, $B C$ $\mathrm{CT}+$ ultrasound, $B D$ radiography + ultrasound, $C \mathrm{CT}$, $D$ radiography. Education level: $A$ illiteracy, $B$ elementary

\section{Clinical Characteristics}

We defined the effectiveness of PRF for ZAP as relief of $>50 \%$ compared to pre-PRF in NRS scores. All participants were divided into positive and negative effectiveness groups. Statistical analysis was performed on continuous and categorical variables, respectively.

For continuous variables, we used the twosample $t$-test to analyze differences between two school level, $C$ junior high school, $D$ senior high school, $E$ university and above. Rash site: $C$ cervical, $H$ head, $L$ lumbosacral, $T$ thoracic. Stage of HZ: A, $\leq 1$ month; B, 1-3 months; C, $>3$ months. Left or right: $L$ left, $R$ right

datasets. Three continuous numeric variables were statistically significant: lymphocyte count in peripheral blood, pregabalin dose, and bodily pain indicators of SF-36 (Fig. 1). The analysis results revealed that lower lymphocyte count in peripheral blood, higher pregabalin dose, and higher bodily pain indicators of SF-36 were significantly associated with poor outcome of PRF on ZAP. 


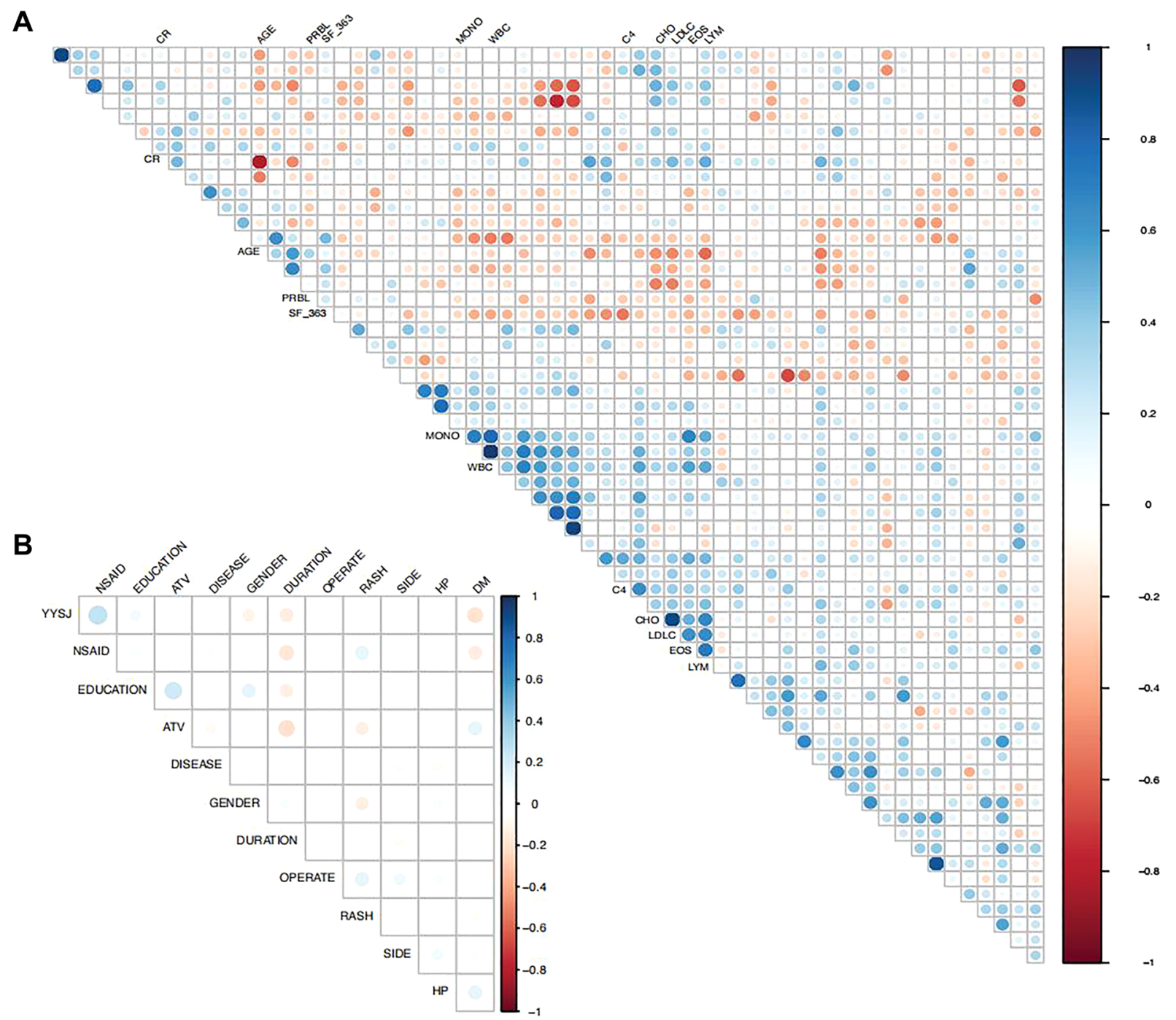

Fig. 3 a Correlation heat map of numerical variables. b Correlation heat map of categorical variables. $C R$ creatinine, $P R B L$ pregabalin dose, $S F-363$ bodily pain indicators of SF-36, $M O N O$ monocytes, $W B C$ white blood cell, $C H O$ cholesterol, $L D L C$ low-density lipoprotein

According to the distribution of categorical variables, sex, guide methods of the procedure, education level, stage of HZ, concomitant disease, side (left/right), and rash site were different in two datasets (Fig. 2). These indicators entered the next step of the correlation analysis before acceptance in the LASSO analysis to determine whether they were related to the effectiveness of PRF therapy in patients with ZAP. cholesterol, EOS eosinophil, LYM lymphocyte, YYSJ neurotrophic drug, $A T V$ antiviral drug, DURATION stage of HZ, OPERATE guiding methods of procedure, RASH rash site, SIDE left or right side of the body, $H P$ hypertension, $D M$ diabetes

\section{Correlation Analysis}

We found that age and eosinophil, monocyte, white blood cell, cholesterol, low-density lipoprotein cholesterol (LDLC), creatinine, and complement C4 levels in peripheral blood correlated significantly with the previously selected variables according to the correlation analysis (Fig. 3). Figure 3a shows a correlation heat map 
A

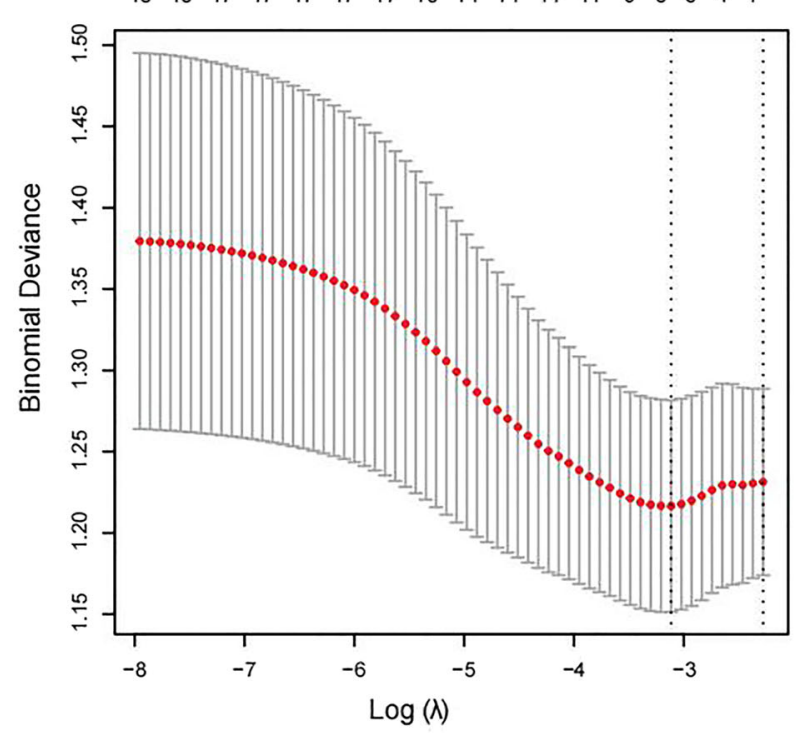

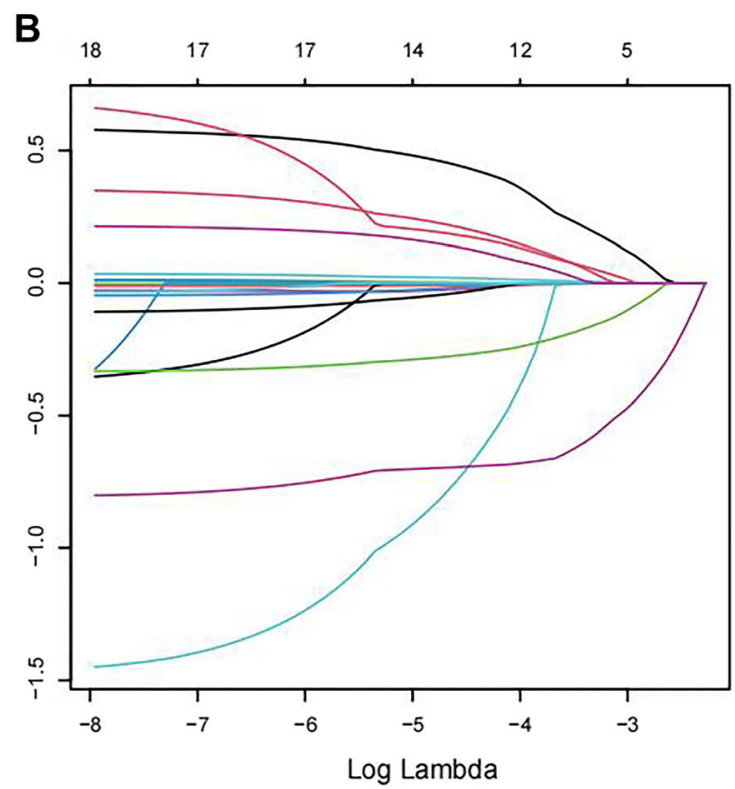

Fig. 4 a Optimal predictor (lambda) selection in the LASSO model. b LASSO coefficient profiles of the factors. Optimal lambda resulted in seven factors with nonzero coefficients (lambda $=0.01594791$ )

Table 3 The regression coefficients of the seven most powerful factors identified by LASSO regression analysis

\begin{tabular}{lc}
\hline Factor & LASSO coefficient \\
\hline Lymphocyte & 0.148 \\
Bodily pain indicators of SF-36 & -0.001 \\
Pregabalin dose & -0.001 \\
LDLC & 0.028 \\
Complement C4 & 0.001 \\
Sex & -0.508 \\
Stage of HZ & 0.128 \\
\hline
\end{tabular}

Model $=1.586+0.148 \times$ lympho-

cyte $+(-0.001) \times$ bodily pain indicators of SF$36+(-0.001) \times$ pregabalin dose $+0.028 \times$ LDLC + $0.001 \times \mathrm{C} 4+(-0.508) \times \operatorname{sex}+(-0.128) \times$ stage of $\mathrm{HZ}$

$L D L C$ low-density lipoprotein cholesterol

of numerical variables, and Fig. $3 \mathrm{~b}$ shows the correlation heat map of categorical variables.

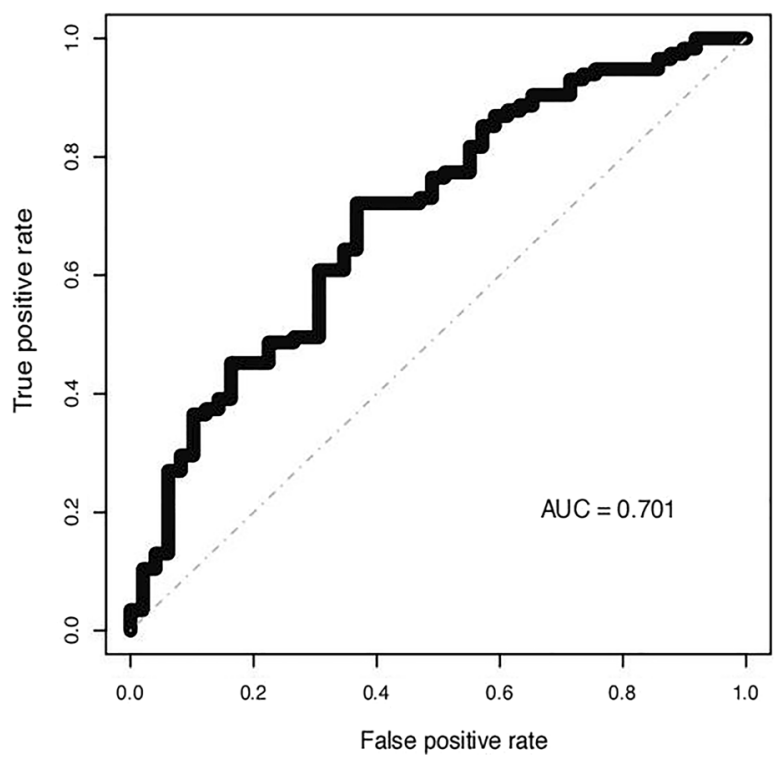

Fig. 5 ROC curve

In summary, 18 variables were selected from all factor-related variables. Finally, we removed samples with missing values in these variables and obtained a total of 164 samples. 


\section{Development of Prediction Model Based on LASSO Regression}

LASSO penalization was used to conduct logistic regression to help reduce the dimensions of feature selection through a tenfold cross-validation for prediction. LASSO regression analysis extracted the seven most powerful features in the prediction model: sex, stage of HZ, pregabalin dose, bodily pain indicators of SF-36, lymphocyte count, and LDLC and complement $\mathrm{C} 4$ in peripheral blood (Fig. 4). Model $=1.586+0.148 \times$ lymphocyte $+(-0.001) \times$ bodily pain indicators of SF-36 $+(-0.001) \times$ pregabalin dose +0.028 $\times$ LDLC $+0.001 \times \mathrm{C} 4+(-0.508) \times \operatorname{sex}+$ $(-0.128) \times$ stage of HZ. The intercept was 1.586 . The regression coefficients of each factor are shown in Table 3 . The prediction for each participant was calculated using the following model equation:

$$
\text { Model }=\beta_{1} X_{1}+\beta_{2} X_{2}+\ldots+\beta_{n} X_{n}+\beta_{0},
$$

where $X$ denotes factors and $\beta$ denotes the regression coefficient of the factors.

\section{Performance of the Prediction Model}

We generated the ROC curve for the prediction model (Fig. 5), which showed an AUC of 0.701. The sensitivity, specificity, and overall accuracy of the model were $90 \%, 33 \%$, and $73 \%$, respectively.

\section{DISCUSSION}

In the current cohort study, our results showed that $69.6 \%$ of patients with ZAP achieved satisfactory improvement after PRF therapy. To clarify the potential predictors of the effectiveness of PRF therapy in patients with ZAP, a statistical model using a LASSO regression was developed. The LASSO analysis revealed seven factors significantly associated with poor PRF outcome: male sex, advanced stage of $\mathrm{HZ}$, higher pregabalin dose, higher bodily pain indicators of SF-36, and lower lymphocyte count, LDLC, and complement C4 in peripheral blood. To the best of our knowledge, this is the first study to investigate multiple identified potential predictors for the effectiveness of PRF therapy.

PHN can adversely affect patients' ability to perform daily self-care tasks and can seriously reduce the quality of life for patients. The damaged sensory neurons in patients with ZAP produce abnormal electric impulses, which are transmitted to the spinal cord and induce spontaneous pain, leading to central sensitization. Regular anti-neuropathic drug administration combined with interventional therapies is widely accepted in ZAP treatment. PRF is easily accepted as an appropriate intervention by both patients and physicians for its proven therapeutic effects, minimal invasiveness, and fewer adverse effects [8]. Although the mechanisms of PRF remain unclear, various researchers have been working toward revealing the underlying processes. Possible mechanisms of PRF action include the induction of long-term depression in the spinal cord that could reversibly disrupt the transmission of impulses across small unmyelinated fibers [9]. Other findings showed that PRF decreased microglial activity in the spinal dorsal horn and reduced pro-inflammatory cytokines such as tumor necrosis factor$\alpha$ and interleukin-6 [10]. However, little has been elucidated about the clinical factors that influence the outcome of PRF therapy. Clinicians should manage therapeutic regimens in accordance with the patient's specific condition and existing measures to achieve personalized treatment.

In our study, among all related predictors, the stage of $\mathrm{HZ}$ was the most important. Although PRF was highly effective for pain reduction in patients with $\mathrm{PHN}$ at all time points, and no study has been conducted to determine optimal timing of PRF use, our results suggest that greater effort should be made to promote PRF to the appropriate patients as early as possible to achieve satisfactory outcomes [1]. Kim et al. [11] reported that the degree of pain reduction was significantly higher in patients who received PRF within 90 days after the herpes phase than in patients who received PRF $>90$ days after the herpes phase. This result indicated that application of PRF to the dorsal root ganglion should be considered as early as 
possible for pain control and prevention of PHN in cases of $\mathrm{HZ}$ that are resistant to conventional medication and blocks. If an appropriate degree of pain reduction is not established in the acute phase of HZ, the risk of PHN development is increased. After managing the pain as quickly as possible, PRF can reduce the economic burden of patients and dose of analgesic drugs.

However, patients with $\mathrm{HZ}$ in the early stage of the disease may visit other departments, such as dermatology, neurology, or traditional Chinese medicine, instead of the department of pain medicine. Although effective treatment is available for some patients, treatment may be delayed for those at high risk of PHN. Increasing the popularization and publicity regarding the department of pain medicine is required so that more patients can obtain timely and effectively individualized treatment plans, which could lead to better pain relief and improve quality of life.

It has been widely accepted that the primary therapy for PHN is early and sufficient administration of medication, including calcium channel regulators (e.g., pregabalin), tricyclic antidepressants (amitriptyline), topical analgesics (5\% lidocaine patch), tramadol, and other opioids [12]. Pregabalin is the most preferred drug in the treatment of ZAP. It was demonstrated that the full dose-response range of pregabalin can balance efficacy and safety to optimize pain relief, global status, and sleep quality [13]. Several retrospective database analyses have reported the administration of low-dose pregabalin in patients with PHN [13]. Physicians must tailor treatment to the individual patient's response. In this study, we found that the dose of pregabalin was associated with the efficacy of PRF therapy. Patients who received a smaller pregabalin dose at admission had greater pain relief after PRF therapy. Low doses of orally administered pregabalin may indicate that the patient has less pain or is at an earlier disease stage, which may be similar to another related indicator, bodily pain indicators of SF-36, which indicates that the lower the pain level, the better the treatment. A systematic review and meta-analysis revealed highquality evidence that severe acute pain was associated with increased risk of PHN and that pain interfering with daily functioning was associated with PHN [14]. All these results indicate that physicians need to inform patients about the complexity of their condition and the importance of obtaining treatment early for better results.

Our study found that a lower peripheral blood lymphocyte level was a negative factor in the effectiveness of treatment, and correlation analysis showed a correlation with LDLC and complement $\mathrm{C} 4$ levels in peripheral blood. This result may provide clues for future individualized treatment.

Many studies have tried to clarify the relationship between $\mathrm{T}$ lymphocyte subset populations and development of PHN. Patients with HIV-1 infection with deficiencies in both CD4+ and CD8+ lymphocytes are at high risk for VZV infection and therefore at higher risk for PHN [15]. The presentation of serum VZV-specific CD4+ lymphocytes was found to decrease with age, and this result suggests that aging people are at elevated risk for developing PHN [16]. An imbalance between Th17 and Treg cells is associated with the reactivation of VZV, which may contribute to the pathogenesis of $\mathrm{HZ}$ [17]. Moreover, a recent study showed a statistically significant increase in helper $\mathrm{T}$ cell ratio (CD3+, CD4+) and Th17 levels before and after treatment, indicating that immunological mechanisms play an essential role in the pathogenesis of neuropathic pain [18]. However, prospective studies should be conducted in the future, as an immunologically based approach will be a critical point in ZAP treatment.

A genome-wide screen in a spinal ligation model of neuropathic pain showed that several complement-related genes, including C1, C3, C4, and C5, were significantly upregulated [19]. PRF that acts to inhibit the complement system may fill major gaps in our study of ZAP mechanisms. Conversely, it was found that hypolipidemic activity could ameliorate the progression of diabetes-induced neuronal impairment and hyperalgesia [20]. The study revealed that LDLC showed a negative correlation with dorsal root ganglion volume, because LDL is required to supply cholesterol to Schwann cells and neurite tips for remyelination after damage to peripheral nerves [21]. It was 
also shown that activation of LDL receptor-related protein 1 (LRP-1) can indirectly reduce neuropathic pain by attenuation of inflammation, reporting better outcomes for neuropathies such as Alzheimer disease, nerve injury, and diabetic peripheral neuropathy. All these results suggest an extremely interesting therapeutic target of the mechanism of PRF on ZAP [22].

The results of a systematic review that characterized the incidence rate of $\mathrm{HZ}$ in the general population showed that the cumulative incidence of $\mathrm{HZ}$ was higher in women than in men worldwide [23]. It was clearly demonstrated that female sex was an independent risk factor for $\mathrm{HZ}$, and women with HZ more frequently had PHN [24]. However, no studies have focused on sex differences in treatment outcomes. In this study, we found that sex was an important factor in PRF effectiveness in patients with ZAP, with women more likely to benefit from ZAP treatment.

One of the limitations of this study is that the data are from a single center, which inevitably limits the results. In the future, more clinical follow-up data can be obtained from multiple medical centers to verify the results of this study. Moreover, the follow-up time of this clinical study was 3 months, and a longer follow-up period is needed to observe the difference in the efficacy of PHN treatment. Moreover, whether the therapeutic effect of PRF can be improved by regulating lymphocytes and other measures needs to be verified. Future studies should address these limitations with well-designed protocols. It is also worth mentioning that a growing body of research suggests that vaccinating individuals at high risk for PHN may be beneficial. If patients with severe immunosuppression are at increased risk of $\mathrm{PHN}$ and at greater risk of $\mathrm{HZ}$ itself, there is even more need to identify alternative strategies to prevent $\mathrm{HZ}$ [25]. For the past 2 years, the HZ vaccine has been officially marketed in China for the prevention of $\mathrm{HZ}$ in adults aged $\geq 50$ years. However, the current use among the population is still limited, and there is a lack of relevant clinical data.

\section{CONCLUSION}

To the best of our knowledge, this is the first study to investigate multiple identified risk factors for the benefits of PRF therapy for the patient with poor responsiveness to drug treatment. Our study found that $69.6 \%$ of patients with ZAP satisfactorily improved after PRF therapy. Furthermore, our study has identified seven factors significantly associated with poor PRF outcome: male sex, advanced stage of HZ, higher pregabalin dose, higher bodily pain indicators of SF-36, and lower lymphocyte count, LDLC and complement C4 in peripheral blood. These results are helpful to optimize the clinical use strategy of PHN and consequently provide valuable recommendations for physicians to make personalized treatment plans.

\section{ACKNOWLEDGEMENTS}

Funding. This research was funded by Zhejiang Province Medicine and Health Major Science and Technology Plan (WKJ-ZJ-2025) and Science Technology Department of Zhejiang Province Plan (2022C03081) and Zhiying Feng funded the journal's Rapid Service Fee.

Authorship. All named authors meet the International Committee of Medical Journal Editors (ICMJE) criteria for authorship for this article, take responsibility for the integrity of the work as a whole, and have given their approval for this version to be published.

Author Contributions. Zhiying Feng conceived and designed the work, developed the study design, was involved with data acquisition and interpretation, drafted the manuscript, edited the draft, and approved the final version. Zhiyou Peng assisted in the data analysis, provided critical review of the manuscript, and approved the final version. Yanfeng Zhang, Xuejiao Guo, Jianguo Guo, Wenguang Huang, Yunze Li, Zhe Yan, Nannan Guo, and Daqiang Ke provided key input into the study design, conduct of research, provided critical review of 
the manuscript, and approved the final version. Jinyan Huang and Li Chen were involved with data analysis, drafted and revised the statistical sections, and approved the final version.

Disclosures. Zhiyou Peng, Jianguo Guo, Yanfeng Zhang, Xuejiao Guo, Wenguang Huang, Yunze Li, Zhe Yan, Nannan Guo, Daqiang Ke, Li Chen, Jinyan Huang, Zhiying Feng have nothing to disclose.

Compliance with Ethics Guidelines. The protocol was approved by the ethics committee of the First Affiliated Hospital, College of Medicine, Zhejiang University, Hangzhou, China (IIT2021116).

Data Availability. The datasets generated during/analyzed during the current study are available from the corresponding author on reasonable request.

Open Access. This article is licensed under a Creative Commons Attribution-NonCommercial 4.0 International License, which permits any non-commercial use, sharing, adaptation, distribution and reproduction in any medium or format, as long as you give appropriate credit to the original author(s) and the source, provide a link to the Creative Commons licence, and indicate if changes were made. The images or other third party material in this article are included in the article's Creative Commons licence, unless indicated otherwise in a credit line to the material. If material is not included in the article's Creative Commons licence and your intended use is not permitted by statutory regulation or exceeds the permitted use, you will need to obtain permission directly from the copyright holder. To view a copy of this licence, visit http://creativecommons.org/licenses/by$\mathrm{nc} / 4.0 /$.

\section{REFERENCES}

1. Wu CY, et al. Efficacy of pulsed radiofrequency in herpetic neuralgia: a meta-analysis of randomized controlled trials. Clin J Pain. 2020;36(11):887-95.
2. Johnson RW, Rice AS. Clinical practice. Postherpetic neuralgia. $\mathrm{N}$ Engl J Med. 2014;371(16): 1526-33.

3. Johnson RW, et al. Postherpetic neuralgia: epidemiology, pathophysiology and management. Expert Rev Neurother. 2007;7(11):1581-95.

4. Yang F, et al. The epidemiology of herpes zoster and postherpetic neuralgia in China: results from a cross-sectional study. Pain Ther. 2019;8(2):249-59.

5. Yu SY, et al. Patient and economic burdens of postherpetic neuralgia in China. Clinicoecon Outcomes Res. 2019;11:539-50.

6. Guo S, et al. The effect of interventional pain management on treating postherpetic neuralgia. Indian J Dermatol. 2019;64(3):251.

7. Wang XX, Zhang Y, Fan BF. Predicting postherpetic neuralgia in patients with herpes zoster by machine learning: a retrospective study. Pain Ther. 2020;9(2):627-35.

8. Wen B, et al. Efficacy of different interventions for the treatment of postherpetic neuralgia: a Bayesian network meta-analysis. J Int Med Res. 2020;48(12): 300060520977416.

9. Shi $\mathrm{Y}, \mathrm{Wu} \mathrm{W}$. Treatment of neuropathic pain using pulsed radiofrequency: a meta-analysis. Pain Physician. 2016;19(7):429-44.

10. Chang MC. Efficacy of pulsed radiofrequency stimulation in patients with peripheral neuropathic pain: a narrative review. Pain Physician. 2018;21(3): E225-34.

11. Kim K, Jo D, Kim E. Pulsed radiofrequency to the dorsal root ganglion in acute herpes zoster and postherpetic neuralgia. Pain Physician. 2017;20(3): E411-8.

12. Hadley GR, et al. Post-herpetic neuralgia: a review. Curr Pain Headache Rep. 2016;20(3):17.

13. Arnold LM, et al. Dose-response of pregabalin for diabetic peripheral neuropathy, postherpetic neuralgia, and fibromyalgia. Postgrad Med. 2017;129(8):921-33.

14. Forbes HJ, et al. A systematic review and metaanalysis of risk factors for postherpetic neuralgia. Pain. 2016;157(1):30-54.

15. Domingo $\mathrm{P}$, et al. Herpes zoster as an immune reconstitution disease after initiation of combination antiretroviral therapy in patients with human immunodeficiency virus type- 1 infection. Am J Med. 2001;110(8):605-9. 
16. Asanuma $\mathrm{H}$, et al. Frequencies of memory $\mathrm{T}$ cells specific for varicella-zoster virus, herpes simplex virus, and cytomegalovirus by intracellular detection of cytokine expression. J Infect Dis. 2000;181(3):859-66.

17. Kim MS, et al. A study of the changes of $\mathrm{T}$ helper 17 cells and regulatory $\mathrm{T}$ cells in herpes zoster. Ann Dermatol. 2017;29(5):578-85.

18. Mercan A, et al. Immunological mechanism of postherpetic neuralgia and effect of pregabalin treatment on the mechanism: a prospective singlearm observational study. Korean J Pain. 2021;34(4): 463-70.

19. Fritzinger DC, Benjamin DE. The complement system in neuropathic and postoperative pain. Open Pain J. 2016;9:26-37.

20. Abo-Salem OM, et al. Beneficial effects of (-)-epigallocatechin-3-O-gallate on diabetic peripheral neuropathy in the rat model. J Biochem Mol Toxicol. 2020;34(8): e22508.
21. Jende JME, et al. Diabetic polyneuropathy is associated with pathomorphological changes in human dorsal root ganglia: a study using $3 \mathrm{~T}$ MR neurography. Front Neurosci. 2020;14: 570744.

22. García-Fernández P, Üçeylera N, Sommer C. From the low-density lipoprotein receptor-related protein 1 to neuropathic pain: a potentially novel target. Pain Rep. 2021;6(1): e898.

23. van Oorschot D, et al. A systematic literature review of herpes zoster incidence worldwide. Hum Vaccin Immunother. 2021;17(6):1714-32.

24. Thompson RR, et al. Herpes zoster and postherpetic neuralgia: changing incidence rates from 1994 to 2018 in the United States. Clin Infect Dis. 2021;73(9):e3210-7.

25. Warrington R, et al. Summary of the NACI update on herpes zoster vaccines. Can Commun Dis Rep. 2018;44(9):220-5. 